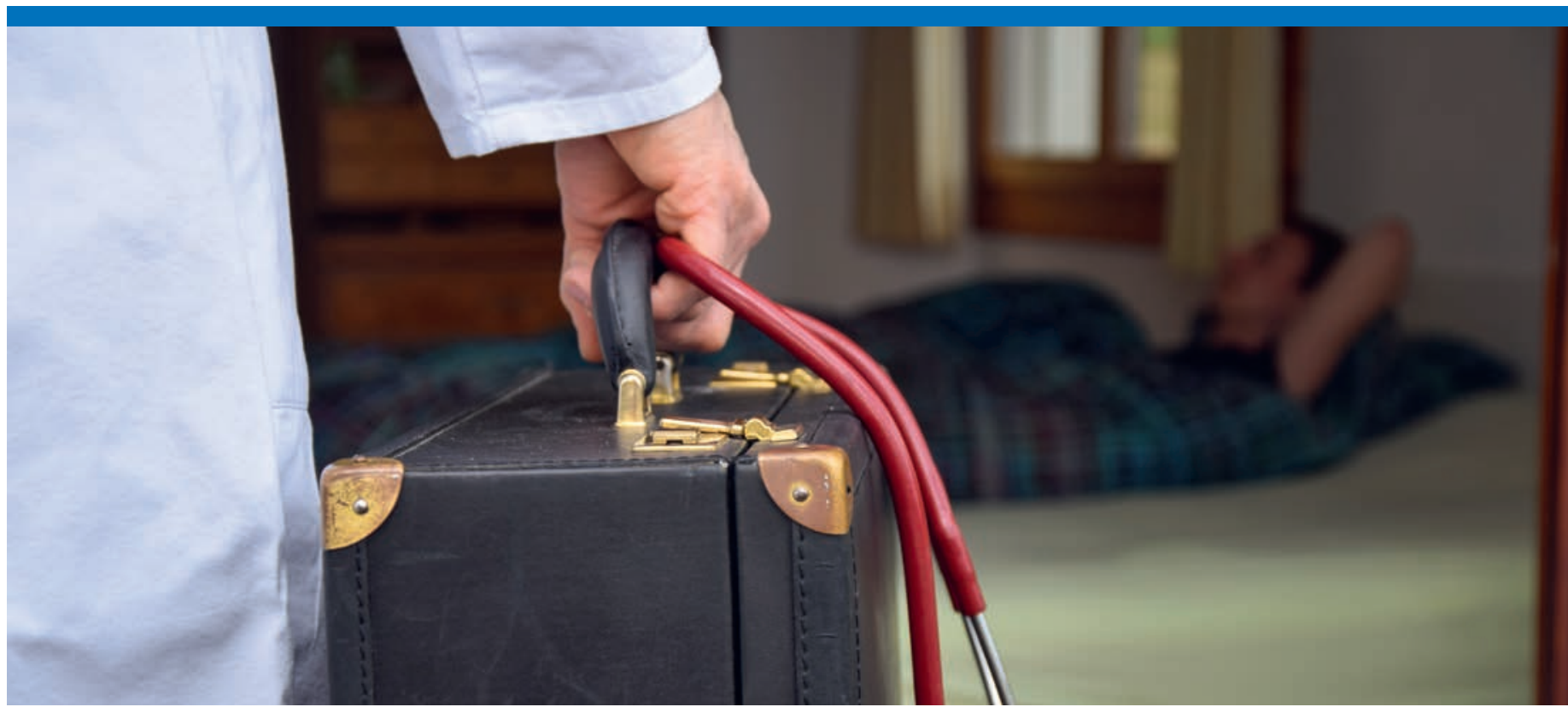

\title{
Als Hausarzt leiste ich Notfalldienst, zumindest im Moment noch ...
}

\section{Beat Stücheli}

Dr. med., SGAIM-Vertreter Plattform Rettungswesen FMH

Ich bin sogenannter Grundversorger. Und nehmen wir mal an, ich bin ein Hausarzt mit Leib und Seele; also ein solcher, der nur das Beste für seine Patienten will. Und dies, so glaubte ich, unterscheidet mich von all den Spezialärzten. Dies ist, so redete ich mir das ein, nämlich «Mein Markenzeichen», worüber ich so stolz bin. Das Markenzeichen der Grundversorger: Der Patient kriegt einen Termin bei mir, wofür und wann immer er ihn braucht. Schlicht eben Kundenfreundlichkeit: «Ich bin stets für Sie da ...»

Doch letzthin traf ich zufällig einen älteren Kollegen, kurz vor der Pensionierung. Es fehle ihm dazu nur noch der oder die geeignete Praxisnachfolgerin. Was ich denn über die Osterfeiertage gemacht hätte, fragte er. Notfalldienst natürlich, was denn sonst, erwiderte ich. Er hatte nur ein mitleidiges Lächeln für mich übrig. Über das sei er längst hinweg, er habe es abdelegieren können. Das sei doch nicht mehr sexy, so gar nicht zeitgemäss ...

Danach drehte es in meinem Kopf: Jedes Jahr werde ich über die Feiertage zum Notfalldienst verknurrt, Tendenz zunehmend. Wieso eigentlich zunehmend? Und ebenso: Jedes Jahr wird der Notfalldienst stressiger, die Patienten anspruchsvoller. Danach bin ich völlig übermüdet, komme gar nicht ausgeruht zurück in den Praxisalltag. Da werde ich gleich von den Ferienrückkehrern bzw. den dadurch erlangten Beschwerden überrollt. Es geht also gleich auf Hochtouren weiter, ein Schrecken ohne Ende ...

Aber warum liegen mir eigentlich die Notfalldienste zunehmend auf dem Magen; warum stossen mir die Notfallpatienten manchmal sauer auf? Werde ich bald zum Misanthrop, und wenn ja, wer trägt die Schuld daran? Bin ich vielleicht einfach frustriert? Irgendwo muss wohl mein Idealismus auf der Strecke geblieben sein. Die Bereitschaft zum Notfalldienst gilt eigentlich in allen Kantonen für Grundversorgerinnen und Grundversorger als Pflicht. Es wird zumeist als zusätzliche Last zum eh schon stressigen Alltagsbetrieb der Praxis empfunden. Beim sich abzeichnenden Hausärztemangel wird die Last auf immer weniger Schultern verteilt. Kein Ende dieses Tunnels ist in Sicht. Das belastet zusehens. Aber warum eigentlich freue ich mich nicht wie jeder Detailhandelsfachmann/jede Detailhandelsfachfrau auf die umsatzreichsten Tage des Jahres? Warum stelle ich mich nicht jetzt bereits auf den Ansturm ein, der jeweils während und nach Weihnachten oder Ostern so sicher kommen wird wie das Amen in der Kirche? Könnte ich vielleicht von den Grundversorgern in den Ski- und Tourismuszentren hierzu etwas lernen? Nun denn, im Moment habe ich keine Zeit, mich solchen Gedanken hinzugeben, denn die Patienten füllen bereits mein Wartezimmer ... 
Es spinnt im Kopf dennoch weiter. Wie ist es zu dieser Misere gekommen, dass wir Grundversorger die Notfallpatienten am liebsten ins Pfefferland wünschen, jede und jeden zusätzlichen als «Was! Der auch noch ...!» empfinden? Habe ich die Geduld und die Kraft verloren und bin somit immer ungeniessbarer geworden für Patienten, für das Praxispersonal und allenfalls auch für meine Familie? Also ist der Idealismus doch dahingeschmolzen?

Was ist überhaupt ein Notfallpatient, wer definiert den Notfallpatienten? Wir Ärztinnen und Ärzte wissen zwar genau, was ein medizinischer, ein traumatologischer Notfall ist. Das haben wir gelernt und ebenso die dazu vorgesehenen Behandlungsstrategien. Aber der Patient selbst will sich irgendwie nicht einfach in dieses Schema hineinpressen lassen. So sucht der Notfallpatient Rat und Hilfe dann, wenn er sich mit seinem eigenen Latein am Ende fühlt, wenn er an seine Schmerzen erinnert wird, wenn der gute Rat der alten erfahrenen Mutter fehlt. Er sucht uns bei allen Tagesund Nachtzeiten auf; nämlich eben dann, wenn er es selbst für notwendig erachtet, wenn er dazu Zeit findet, wenn ihn Dr. Google mit Schreckensszenarien zu sehr verunsichert hat.

Dies spüren Notfallstationen schon lange mit ihren sogenannten Walk-in-Patienten. Viele Notfallstationen von Regional- und Kantonsspitälern haben sich diesem Wandel angepasst, anpassen müssen. Anstatt Patienten abzuwehren, hat man in Form von Triagestellen (telefonisch oder direkt vor Ort) und Notfallpraxen die Bagatell-Notfälle von den (aus Ärztesicht) tatsächlichen Notfällen zu separieren versucht. Dies gelingt mittlerweile vielerorts recht gut.

In städtischen Agglomerationen sind professionelle Organisationen entstanden, welche den Patienten genau den bei Grundversorgern immer unbeliebteren Hausbesuch auch nachts und am Wochenende anbieten und offensichtlich auch zumeist prompt und kundenfreundlich bewerkstelligen. Diese Marktlücke füllt sich offensichtlich von selbst, ohne dass dies irgendwelche Direktiven von oben veranlasst hätten.

Und wie sieht es in der Peripherie aus, wo neben Arztpraxen auch Volg-Lädeli und Postschalter verschwinden? Dort verlangt man von den Patienten, dass sie selbst mobil bleiben und den Weg ins Zentrum, also ins Regionalspital, auf die nächste Notfallstation oder Permanence finden müssen. Die meisten Patientinnen und Patienten sind anscheinend auch dazu bereit. Verlierer dieses Systems bleiben die älteren Menschen, welche kein eigenes Auto haben und auch nur bedingt die öffentlichen Verkehrsmittel in Anspruch nehmen können. Wer hilft denn diesen? Sind wir, das heisst die letzten verbleibenden Grundversorgerin- nen und Grundversorger, für diese Vergessenen des ärztlichen Notfalldienstes verantwortlich? Oder etwa die Politiker? Könnten wir da vielleicht das Konzept der Kantone, wenn es so eines gibt, zur Erhaltung der Posthalterstellen und Volg-Lädeli in der Peripherie 1:1 übernehmen? Oder gäbe es eine andere Strategie? Irgendwie werde ich den Eindruck nicht los, dass man vielerorts in der Notfalldienstversorgung nicht mehr agiert, sondern nur noch reagiert. Es fehlt eine vorausschauende Strategie. Genau so, wie ich es nicht schaffe, mich für den nächsten Weihnachtsdienst zu wappnen und wiederum überrollt werde.

Wie soll man der stetig steigenden Anzahl von Sofortkonsultationen zu allen Tages- und Nachtzeiten begegnen, wie geht diese Entwicklung weiter? Besteht ein Handlungsbedarf, um auch in Zukunft diese Patientenströme bewältigen zu können und dennoch die Kundenfreundlichkeit zu wahren? Von politischer Seite her scheint man zwar das Problem erkannt zu haben, steht ihm aber vielleicht etwas ratlos gegenüber. Erfolglos wird versucht, mit irgendwelchen Zusatzgebühren Patientinnen und Patienten strafend zu erziehen. Dieser Kampf ist hoffnungslos. Patienten fühlen sich zu Unrecht bestraft: Man hat sich ja genau jetzt als Notfall gefühlt, weswegen man halt spätnachts mit seinen über Tage anhaltenden Rückenschmerzen nun endlich Linderung sucht. Und nicht zu vergessen: Irgendwann werde ich mich ja selbst auch als solcher Notfall fühlen und bin dann auch froh, rasch, kompetent und umfassend und erst noch freundlich Hilfe zu erhalten. Notfallpatienten wird es immer geben. Tendenz offensichtlich steigend. Ist dies nun eine Plage, eine Volksseuche oder eine Realität, der wir mit professioneller Kundenfreundlichkeit begegnen wollen?

Irgendwie bin ich es müde, wie offensichtlich viele meiner gleichaltrigen Kolleginnen und Kollegen auch, allzeit bereit zu sein für die dringenden Anliegen und Sorgen meiner Kunden. Vermutlich gibt es bald auch professionellere Notfalldienstorganisationen als mich. Es regelt sich vielleicht ja von selbst. So wie bei meinem bald pensionierter Berufskollegen, der keinen Notfalldienst mehr leisten muss. Ist nun er oder ich zeitgemässer?

Ist nun etwa damit sogar das eingangs erwähnte «Mein Markenzeichen», worauf ich so stolz war, verloren gegangen?

Nein, nein, genug Gedanken gesponnen! Ich bin immer noch mit Leib und Seele Hausarzt. Und stolz darauf, auch wenn ich, von den wenigen verbleibenden Mitkämpferinnen und Mitkämpfern abgesehen, bald einmal der einzige Notfalldiensttuende sein werde ...

Bildnachweis Beat Stücheli 